\title{
Power Model of Impulse Arc Discharge Generation in Active Media Optical Pumping Xenon Lamps of Solid-State Lasers
}

\author{
Yury Anatolievitch Mandryko \\ ITMO University \\ St.-Petersburg \\ mandryko1@rambler.ru
}

\author{
Aleksander Sergeevitch Chirtsov \\ Saint-Petersburg Electrotechnical Uni- \\ versity ETU "LETI" \\ Herzen State Pedagogical University of \\ Russia \\ St.-Petersburg \\ alex chirtsov@mail.ru
}

\author{
Vladimir Mikhailovitch Mikushev \\ Saint-Petersburg State University \\ St.-Petersburg \\ v.mikushev@spbu.ru
}

\begin{abstract}
The semi-empirical model of arc impulse discharge development in impulse xenon lamps for optical pumping of solid-state lasers' active media was considered. Equations of the power balance of supplied electrical power and processes of ionization, plasma heating, heat dissipation and optical radiation generation laid the model's basis. The analytical description of the processes being considered results in an ordinary differential equation which allows a non-resource-intensive numerical solution. The built model enables to evaluate basic electrical and optical parameters of non-local arc discharge plasma at its development stage. Comparison with the results of measurements of electrical and optical plasma parameters which are the most convenient for experimental diagnostics in the modelled non-stationary mode testifies to adequacy of the developed model. Relative simplicity and convenience of the model predetermines its possible usage when solving applied engineering tasks of optimization of operating parameters of impulse pumping lamps and for obtaining initial (zero) approximations for comprehensive modelling of a non-local non-stationary gas discharge plasma in science-intensive calculations taking into account a great combination of elementary collision and radiation processes in a non-equilibrium system.
\end{abstract}

Keywords - expanded xenon non-equilibrium plasma channel, impulse direct xenon lamp.

\section{INTRODUCTION}

Current development of technical means of numerical modelling of complex physical systems predetermined emergence of a new low-temperature plasma physics development stage. Emergence of the non-local plasma model [1] at the beginning of a new century and fast popularity growth during description of gas discharge media predetermined quick development of a new low-plasma physics area connected with macroscopic averaged numerical modelling of a large combination of elementary collision and radiation processes as part of hydrodynamically similar approximations [2]. A large number of works dedicated to such an approach during the study of quite a wide set of diverse gas discharge media (for example, [3--7]) enables to formulate a number of major problems which occurred in this area.
First of all, they include difficulties of restriction of multiply elementary plasma processes [8] taken into consideration when developing quasi-closed models based on the set of reliable data on their probabilistic characteristics [9] or systematically made quantummechanical calculations, resource-intensity of which can be mitigated significantly by means of transitions to different semi-empirical approximations [10, 11]; yet limited experience of comparison of plasma modelling results with the experiment [12]. In this regard, it appears to be expedient to fulfil a series of works for modelling of non-local plasma media of already used or developed technical devices with a view to check the quality of developed theoretical approaches in the non-local plasma physics [13], on the one hand, and to solve applied tasks of optimization of real devices on the other hand [14 - 16]. The practice of numerical modelling of non-local plasma in a quite popular today medium Comsol [17] shows that the quality and speed of models' convergence largely depend on selection of initial (zero) approximations for non-local plasma parameters, and it turns out to be particularly significant during the analysis of non-stationary processes quickly developed in time [18]. In this respect, the present work was made for development and testing of a simple semi-empirical model for the calculation of obtaining a possibly more qualitative approximation for internal (hardly susceptible to experimental determination) parameters of plasma of the developed discharge in xenon which emerges during generation of impulse arc discharges in xenon pumping lamps used in solid-state lasers.

Pulse stroboscopic direct xenon lamps ISP-200, ISP-250, ISP-600, ISP-2000, ISP-2500, INP-5/75, INP-5/40, INP-5,5/80, INP-7/80, INP-5,5/60) and their

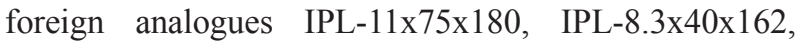
IPL-11x80x180, IPL-14x80x300, IPL-10.5x60x182 and others are widely used: in impulse light sources $[19,20]$, in solid-state and chemical lasers [21-23], in state-of-the-art electrophysical units, for distance measurement, in alarm, in high-speed photography, for 
welding and cutting, in medicine, cosmetology, light engineering, photochemistry, spectrophotometry, water, air decontamination technologies, medications, solutions, instruments, food products, packing materials [24-34]. A wide spectrum of fields of application of impulse lamps predetermines the need for variation of their radiation parameters. These parameters depend on the dynamics of generation and expansion of an arc discharge plasma channel and, hence, can be adjusted within given limits by varying electrical power modes. For the efficient selection of such modes which assures optimization of optical characteristics, it is expedient to develop abovementioned semi-empirical models interconnecting external, easily changing in practice, and important for optimum operation internal electrical and optical characteristics of impulse arc discharge non-local plasma. The specified considerations predetermined the choice of impulse xenon gas discharge lamps as an object of study, the first stage of which the present work is dedicated, the objectives of which include:

- development of a macroscopic model initial for microplasma modelling, on the basis of power balance equations for a gas discharge cell in each moment of a channel expansion stage;

- comparison of calculation results with the experiment for electric power supplied in a gas discharge lamp and power of radiation generated by a lamp at the quasi-stationary plasma channel generation stage;

- development of theoretical assessments for internal non-stationary non-equilibrium process parameters: dependences on time of radiation power of an expanded xenon non-equilibrium plasma channel, mean xenon plasma radiation absorption coefficient, power losses for gas ionization, plasma heating in the developed channel, heat transfer in gas surrounding a plasma channel.

- development of semi-empirical data on relations between listed non-stationary arc discharge parameters.

\section{SEMI-EMPIRICAL MODEL OF THE PROCESS OF IMPULSE} ARC DISCHARGE DEVELOPMENT IN XENON

During the development of the model of arc discharge development processes in impulse xenon lamps, the equation of the power balance at the non-equilibrium plasma channel expansion stage was used. The electrical field power $N_{\Sigma}$ supplied per a unit of time in a discharge gap is consumed for ionization of inert gas atoms $\left(N_{\text {ion }}\right)$ heating of generated plasma $\left(N_{\mathrm{T}+}\right)$, heat transfer in surrounding non-ionized gas $\left(N_{\mathrm{T}}\right)$, and for generation of electromagnetic radiation $\left(\Phi_{\mathrm{rad}}\right)$

$$
N_{\Sigma}=N_{\text {ion }}+N_{\text {T+ }}+N_{\mathrm{N}-}+\Phi_{\text {rad }} .
$$

On the other hand, the power described by the sum (1) can be experimentally determined by a voltage drop in the discharge gap $u$ and the value of discharge current I. The electric power dissipated on a positive discharge column $N_{+}$may be found by discharge current and specific plasma conductivity $\sigma$ in this discharge field.

The speed of system power loss for radiation of electromagnetic waves $\Phi_{\text {rad }}$ through the side surface S of expanded plasma channel, which is included in (1) and is of greatest practical interest, may be determined by plasma radiation spectral density $E_{v, \mathrm{~T}}$ at effective radiating medium temperature:

$$
\Phi_{\mathrm{rad}}=2 \pi S \int_{0}^{\infty} E_{\mathrm{v}, \mathrm{T}} d v
$$

Function $E_{v, T}$ standing under integral (2) may be evaluated by the Planck formula for equilibrium radiation spectral density and Kirchhoff optical law connecting radiating capacity $\left(E_{v, \mathrm{~T}}\right)$ and absorbing capacity of the "grey" body $A_{v, \mathrm{~T}}$ :

$$
E_{\mathrm{i}, \mathrm{T}} / A_{\mathrm{v}, \mathrm{T}}=\text { const. }
$$

Herewith in evaluation calculations based on the Planck formula of radiation of significantly nonequilibrium (at the arc discharge development stage) medium characterized by different electrons and heavy particles temperatures it is reasonable to use the typical electron kinetic power $T_{e}$ as effective temperature.

As a result, the expression for plasma channel radiation power $\Phi_{\text {rad }}$ follows from (2) and (3):

$$
\Phi_{\mathrm{rad}}=\frac{4 \pi}{c^{2}} \mathrm{~h}\left(\frac{\mathrm{h}}{k T}\right)^{3} S \int_{0}^{\infty} A_{v, \mathrm{~T}} \frac{\omega^{3}}{\exp \left(\frac{\mathrm{h} \omega}{k T}\right)-1} d \omega
$$

The experimentally observed lamp radiation power $\Phi_{\text {lamp }}$ differs from $\Phi_{\text {rad }}$ due to partial light absorption by bulb walls. In a restricted spectral frequency range from $v_{1}$ to $v_{2}$ corresponding to the bulb clarity band, the specified effect can be taken into consideration [24] by introducing effective (for a frequency band corresponding to the lamp bulb glass pass band) quartz wall radiation transmission coefficient, $\langle\gamma\rangle$. Its inclusion in (4) where an integration variable is replaced with a dimensionless value $x=h v / k T$ results in the following expression for the impulse lamp light flow measured during the experiment:

$$
\Phi_{\text {lamp }}=\frac{4 \pi}{c^{2}} k T_{\mathrm{e}} S\langle\gamma\rangle \int_{h c /\left(k T_{\mathrm{e}} \lambda_{2}\right)}^{h c /\left(k T_{\mathrm{e}} \lambda_{1}\right)} A_{\mathrm{x}, \mathrm{T}} \frac{x^{3}}{\exp (x)-1} d x
$$

For evaluation of the mean value of plasma channel absorption coefficient $A_{v, T}$ included in (8) the approximate approach based on the Bouguer law was used in [24]:

$$
\langle A\rangle \approx 1-\exp (-K r)
$$

where $K\left[\mathrm{~m}^{-1}\right]$ - mean by spectral interval xenon plasma radiation absorption value, $r$ - plasma channel radius. In its turn, the mean xenon plasma radiation absorption value $k$ included in (6) was evaluated based on classical considerations (Schirmerformula [20]):

$$
K \approx B_{1} \frac{p}{T_{\mathrm{e}}^{3}} \exp \left(-\frac{e U_{\mathrm{i}}}{k T_{\mathrm{e}}}\right)
$$

where $B_{1}=7.9 \cdot 10^{13} \mathrm{~K}^{3} /(\mathrm{Pa} \mathrm{m}), \mathrm{p}$-xenon pressure in a plasma channel, $U_{\mathrm{i}}=11.7 \mathrm{~B}-$ xenon atom ionization potential, $e$ - elementary charge. For the calculation of thermodynamic pressure of xenon plasma channel with volume $V$ Van der Waals equation for xenon was used. As a result of insertion of Ratios (6) and (7) in (5) there 
appears an approximation convenient for its quantitative evaluations for the effective absorption coefficient:

$$
\langle A\rangle \approx 1-\exp \left[B_{1} \frac{\rho R T_{\mathrm{i}} r}{\left(\mu-b_{\mathrm{Kr}} \rho\right) T_{\mathrm{e}}^{3}} \exp \left(-\frac{e U_{\mathrm{i}}}{k T_{\mathrm{e}}}\right)\right]
$$

included in final expressions for electromagnetic radiation flows by a plasma channel and impulse lamp:

$$
\begin{gathered}
\Phi_{\mathrm{rad}}=\frac{4 \pi h}{c^{2}}\langle A\rangle\left(\frac{k T_{\mathrm{t}}}{h}\right) S \int_{0}^{\infty} \frac{x^{3}}{\exp (x)-1} d x \\
\Phi_{\text {lamp }}=\langle\gamma\rangle \frac{4 \pi h}{c^{2}}\langle A\rangle\left(\frac{k T_{\mathrm{e}}}{h}\right) S \int_{h c /\left(k T_{\mathrm{e}} \lambda_{2}\right)}^{h c /\left(k T_{\mathrm{e}} \lambda_{1}\right)} \frac{x^{3}}{\exp (x)-1} d x
\end{gathered}
$$

Final expressions formulas for calculations (8-10) introduce the following designations:, $R$ - universal gas constant, $T_{\mathrm{i}}$ - plasma channel ion temperature, $a_{\mathrm{kr}}, b_{\mathrm{kr}}-$ critical constants for xenon, $P_{\mathrm{kr}}=5.84$ - critical xenon pressure and $T_{\mathrm{kr}}=289.734 \mathrm{~K}$ - critical xenon temperature, $\rho$ - gas density, $\mu$ - its molecular weight. Quartz wall pass band wave lengths $\left(\lambda_{1}=180 \mathrm{~nm}\right.$ to $\left.\lambda_{2}=1100 \mathrm{~nm}\right)$ for lamp types IPL-8.3x40x162, INP-5/40 and ISP250 and the effective radiation transmission coefficient $(<\gamma>)$ are given in $[20,36]$.

For accounting power losses $N_{\mathrm{T}+}$ spent for heating a gas component from heavy particles (ions in atoms) in the expanded plasma channel it was taken into account that density $(\rho)$ and specific heat intensity (c) of plasma at its expansion stage vary weakly as compared to its volume $(V)$ and ion temperature $\left(T_{\mathrm{i}}\right)$ :

$$
N_{\mathrm{T}+}=\frac{d}{d t} c m T_{\mathrm{i}}=\rho c \frac{d}{d t}\left(V T_{\mathrm{i}}\right)=\rho c\left(V \frac{d T_{\mathrm{i}}}{d t}+T_{\mathrm{i}} \frac{d V}{d t}\right)
$$

For exclusion of ion temperature included in (11) the Shockley formula typical for the classical gas discharge thermal physics connecting the volume variation speed and plasma channel temperature was used:

$$
\frac{d T_{\mathrm{i}}}{d t}=\frac{1}{\alpha V} \frac{d V}{d t}, \quad T_{\mathrm{i}}-T_{\mathrm{io}}=\frac{1}{\alpha} \ln \frac{V}{V_{0}}
$$

where $\alpha_{\mathrm{kr}}=1.087 \cdot 10^{-3} \mathrm{~K}^{-1}$ - plasma channel volumetric expansion coefficient. Its applicability amidst developed arc discharge was checked based on experimentally obtained dependence of a plasma channel proportional to the cross section area (and, hence, its volume) of discharge current on temperature. Applicability of the relation (12) between the plasma channel volume and temperature for the considered discharge development stage was checked experimentally.

Sizes, volume of a plasma channel and their expansion speed using the Ohm's law recorded in a differential form are easily expressed via electrical discharge parameters convenient for measurement: specific electrical conductivity of plasma channel $\sigma$, its variation speed in a positive column with the length of $\mathrm{H}$ and current intensity through a plasma interval i. As a result of insertion in (11) of so transformed ratios (12), the relation between heat power $N_{\mathrm{T}+}$ and electrical discharge gap parameters convenient for comparison with the experiment is obtained:

$$
N_{\mathrm{T}+}=\rho c T_{\mathrm{i}} \frac{H^{2}}{\sigma} \frac{d y}{d t}, \quad T_{\mathrm{i}}=T_{0}+\frac{1+\ln \left(i / I_{0}\right)}{\alpha}
$$

The ratio (13) via $I_{0}$ and $T_{0}$ designates initial (i.e. established during the time of combustion of a pilot arc preceding voltage power pulse arrival) current and ion temperature values of a plasma channel, respectively. Specific conductivity of a positive column is determined by electronic temperature $\sigma=D\left(T_{\mathrm{e}}\right)^{2}$ where $D_{1}=3.2 \cdot 10^{-}$ ${ }^{5} \mathrm{Siemens} /\left(\mathrm{m} \cdot \mathrm{K}^{2}\right)$ [24]. The latter at the plasma channel expansion stage can be deemed approximately invariable [24].

Power losses $N_{\text {ion }}$ required for providing generation and expansion of a non-equilibrium plasma channel are related to its volume variation speed $V$ through multiplication of charged particles concentration in plasma $n_{\mathrm{e}}$ by power $w_{\mathrm{i}}$ required for ionization of one atom with generation of an electron with power corresponding to electronic temperature of a xenon plasma ("ionization specific power"):

$$
N_{\text {ion }}=\frac{d\left(w_{\mathrm{i}} n_{\mathrm{e}} V\right)}{d t} \approx w_{\mathrm{i}} n_{\mathrm{e}} \frac{d V}{d t}, \quad w_{\mathrm{i}}=\left(U_{\mathrm{i}}+\frac{3}{2} k T_{\mathrm{e} 0}\right)
$$

The formula for the calculation of the heat dissipation power $N_{\text {T- }}$ from the expanded xenon non-equilibrium plasma channel with time variable ion temperature $T_{\mathrm{i}}$ in the environment at temperature $T_{0}=300 \mathrm{~K}$ through the boundary area with $\Delta l$ width has the following form:

$$
N_{\mathrm{T}-}=\chi S \frac{T_{\mathrm{i}}-T_{0}}{\Delta l}
$$

where $\chi=3 \cdot 10^{-2} \cdot \mathrm{W} /(\mathrm{m} \cdot \mathrm{K})$ - xenon heat conductivity coefficient. The side surface area $S$ and radius $r$ of the expanded plasma channel are determined via discharge current I using the differential Ohm's law:

$$
r=\sqrt{\frac{H I}{\pi \sigma\left(u_{1}-U_{\mathrm{a}}-U_{\mathrm{k}}\right)}}, \quad S=2 \pi r H
$$

Values of near-electrode potential drops $U$ and $U_{\mathrm{k}}$ included in (16) in total do not exceed several volts and are low as compared to voltage drop $\mathrm{u}_{1}$ on a positive column of approximately $40 \mathrm{~V}$ in the pilot arc mode and reaching $400 \mathrm{~V}$ in the power impulse mode.

Use of (16) for expression of geometrical parameters of the expanded plasma channel included in (14) and (15) via discharge current intensity and plasma channel conductivity easily measured at the experiment $y=\sigma \mathrm{s} / L$ enables to obtain the ultimate working formula for calculation of total heat losses and to transform using (4) the initial equation (1) into the differential equation for finding a dependence of current intensity on time:

$$
\begin{aligned}
& I^{2} \sigma^{-1}=\left\{\rho c\left[T_{0}+\frac{1+\ln \left(I / I_{0}\right)}{\alpha}\right]+n_{\mathrm{e}}\left(U_{\mathrm{i}}+\frac{3}{2} k T_{\mathrm{e} 0}\right)\right\} \frac{H^{2}}{\sigma} \frac{d y}{d t}+ \\
& +\frac{4 \pi h}{c^{2}}\langle A\rangle\left(\frac{k T_{\mathrm{t}}}{h}\right) S \int_{0}^{\infty} \frac{x^{3}}{\exp (x)-1} d x+\chi S \frac{T_{\mathrm{i}}-T_{0}}{\Delta l}
\end{aligned}
$$

\section{Discharge Numerical Modelling}

Equations obtained in the considered power model make up the closed system which enables to calculate 
the course of dependences of discharge current on time $i_{1}(t)$ and other systems characteristics at the nonequilibrium plasma channel expansion stage as part of made approximations.

The built differential equation (17) was solved in the course of its numerical integration for discharge current, via which conductivity $\mathrm{y}$, ion temperature $\mathrm{T}_{\mathrm{i}}$, mean absorption coefficient $\langle A\rangle$, plasma channel side surface area $\mathrm{S}$ were expressed. The integration step did not exceed one hundredth of typical time of the aggregate arc discharge generation process.

Calculations were performed for the case of the value of constant voltage drop between lamp anode and cathode $u_{1} \approx$ const, which, in its turn, implied invariability of the potential difference between positive column edges. This feature enables to simplify the equation (17) by transferring in it from conductivity to voltage drop on a positive column $U_{\text {colomb }}$ :

$$
\begin{aligned}
& i_{1} U_{c o l o m b}=\left\{\rho c\left[T_{0}+\frac{1+\ln \left(I / I_{0}\right)}{\alpha}\right]+n_{\mathrm{e}}\left(U_{\mathrm{i}}+\frac{3}{2} k T_{\mathrm{e} 0}\right)\right\} \times \\
& \frac{H^{2}}{\sigma} \frac{d y}{d t}+\frac{4 \pi h}{c^{2}}\langle A\rangle\left(\frac{k T_{\mathrm{t}}}{h}\right) S \int_{0}^{\infty} \frac{x^{3}}{\exp (x)-1} d x+\chi S \frac{T_{\mathrm{i}}-T_{0}}{\Delta l}
\end{aligned}
$$

As a result of solution of the power balance equation (18) the time behaviour of the instant current value $I(t)$ of pumping in a discharge lamp was calculated, by means of which time dependences of instant electric power supplied in lamp and its instant radiation power were calculated. Thus, the obtained modelling results were compared with an experiment.

\section{EXPERIMENT}

The experimental test of the built model was made on lamp types IPL-8.3x40x162 ISP250 for two voltage values at storage capacity $\left(U_{\mathrm{la} 1}=80 \mathrm{~V}\right.$ and $\left.U_{\mathrm{la} 2}=110 \mathrm{~V}\right)$ [37].

Recording of a transitional instant voltage characteristic proportional to instant pumping voltage between electrodes. Recording of the discharge current time behaviour in an impulse lamp is carried out using non-inductive current sensor.

Figure 1 shows experimental and design transitional characteristics of electric powers $N_{\mathrm{el}}(t)$ supplied in the expanded xenon non-equilibrium plasma channel in INPKL (ISPKL).

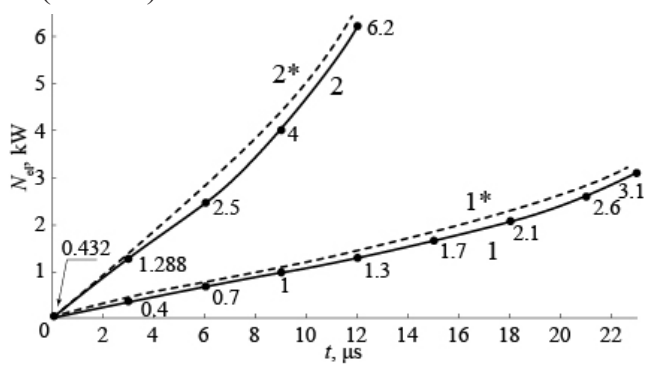

Fig. 1. Transitional characteristics of electric powers supplied in a xenon lamp: 1 and $1 *$ _ experimental and calculated characteristics for $80 \mathrm{~V}$ and $140 \mathrm{~V}$ supply voltage, 2 and $2 *$ — experimental and calculated characteristics for the same voltages.

As it follows from figure 2, calculated and experimental transitional characteristics $N_{\mathrm{el}}(t)$ during expansion of a xenon non-equilibrium plasma channel rise and match satisfactorily. It testifies in favour of the proposed physical and mathematical model.
Figure 2 shows design time dependences of common logarithms of rated powers for ionization of an expanded plasma channel $\lg \left(N^{(\mathrm{i})}{ }_{\text {channel }}(t) / N^{(\mathrm{e})}{ }_{\text {pdao }}\right)$ (8), for heating a plasma channel $\lg \left(N^{(\mathrm{T})}{ }^{\text {channel }}(t) / N^{(\mathrm{e})}{ }^{\mathrm{pda0}}\right)(13)$, for radiation of a plasma channel $\lg \left(N^{(\mathrm{r})}\right.$ dissipation from channel $\lg \left(N^{(x)}(t) /{ }_{\mathrm{xe}}^{\mathrm{pdao}} N^{(\mathrm{e})}{ }_{\mathrm{pdaa}}\right)$ (27) in respect of electric power supplied in a plasma channel of a pseudo-pilot arc.

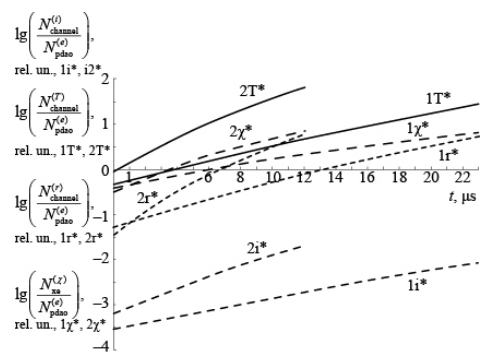

Fig. 2. Timedependences of common logarithms of rated powers for ionization of an expanded plasma channel $\lg \left(N^{(\mathrm{i})}{ }(t) / N^{(\mathrm{e})}\right)$, for heating a plasma channel $\lg \left(N^{(\mathrm{T})}{ }_{\text {channel }}(t) / N^{(\mathrm{e})}{ }_{\text {pdao }}\right)$, radiation of a plasma channel $\lg \left(N^{(\mathrm{r})}{ }_{\text {channel }}(t) / N^{(\mathrm{e})}{ }_{\text {dao }}\right)$ and heat dissipation from it into the environment $\lg \left(N^{(x)}(t) / N^{(\mathrm{e})}{ }^{\mathrm{d}}\right)$ in respect of electric power supplied in a plasma channel of a pseudo-pilot arc: $1 i^{*}, 2 i^{*}$ — calculated characteristics for powers of plasma channel ionization at supply voltage values on a lamp of $80 \mathrm{~V}$ and $110 \mathrm{~V}$ respectively, 1T*, 2T* - calculated characteristics for powers of expanded plasma channel heating at supply voltage values on a lamp of $80 \mathrm{~V}$ and $110 \mathrm{~V}$, respectively; $1 \mathrm{r}^{*}$, $2 \mathrm{r}^{*}$ - calculated characteristics for plasma channel radiation powers at supply voltage values on a lamp of $80 \mathrm{~V}$ and $110 \mathrm{~V}$, respectively; $1 \chi^{*}, 2 \chi^{*}$ — calculated characteristics for heat dissipation powers from a channel into the environment at supply voltage values on a lamp of $80 \mathrm{~V}$ and $110 \mathrm{~V}$, respectively.

As one could expect, at the xenon non-equilibrium plasma channel expansion stage time dependences of common logarithms of rated powers for ionization of an expanded plasma channel $\lg \left(N^{(\mathrm{i})}{ }_{\text {channel }}(t) / N^{(\mathrm{e})}{ }_{\text {pdao }}\right)$, for heating a plasma channel $\lg \left(N^{(\mathrm{T})}{ }_{\text {channel }}^{\text {channel }}(t) / N^{(\mathrm{e})}{ }_{\text {pdao }}^{\text {pdao }}\right)$, for radiation of a plasma channel $\lg \left(N^{\mathrm{rr})}{ }_{\text {channel }}^{\text {channel }}(t) / N^{\mathrm{pe})}{ }_{\mathrm{pdao}}^{\mathrm{pda}}\right)$ and heat dissipation from a channel into the environment $\lg \left(N_{\mathrm{xe}}^{(x)}(t) / N_{\text {pdao }}^{(\mathrm{e})}\right)$ are increased with time at the same lamp voltage value. Herewith, voltage increase between lamp electrodes results in an increase of rates of power rise for ionization of an expanded plasma channel, for heating a plasma channel, for radiation of a plasma channel and heat dissipation from it into the environment.

Recording of a transitional characteristic of a radiation power surface density INPKL (ISPKL) is made using VD3 photodetector and $\mathrm{R}_{4}$ current sensor. Based on the experimental transitional characteristic of radiation power surface density INPKL (ISPKL) experimental transitional characteristic of radiation power INPKL (ISPKL) $N_{\mathrm{ijl}}(t)$ was determined. The latter is given in figure 3 together with numerical modelling results.

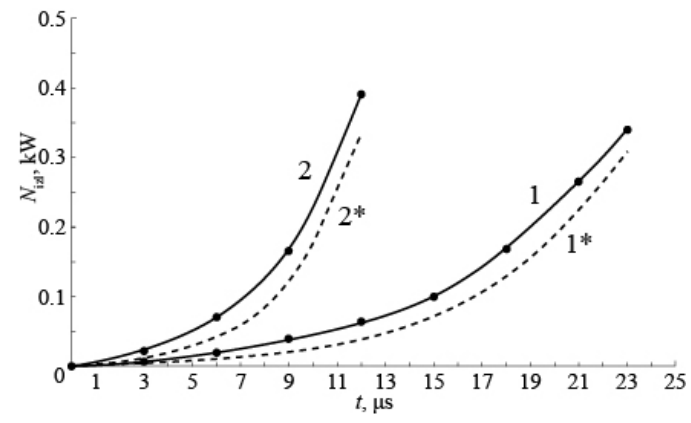

Fig. 3. Transitional characteristics of radiation powers INPKL (ISPKL): 1 and $1 *$ _experimental and calculated characteristics for $80 \mathrm{~V}$ and $140 \mathrm{~V}$ supply voltage, 2 and $2 *$ - experimental and calculated characteristics for the same voltages. 
Satisfactory matching of numerical modelling results with the experiment enables to speak about applicability of the proposed model for rough evaluations of the time course of parameters of non-equilibrium plasma of an impulse arc discharge in xenon pumping lamps at the plasma channel expansion stage.

Development of the numerical expanded plasma channel model based on power balance accounting enables not only to obtain initial evaluations for fullscale plasma modelling taking into account specifics of elementary collision and radiation plasma processes significant for the considered system but also gives a possibility to evaluate a number of internal parameters of non-local non-stationary expanded discharge channel plasma important for optimization of pumping lamps operation.

\section{Conclusion}

As a result of theoretical and experimental studies, a model for processes of expanded non-equilibrium plasma channel generation in impulse stroboscopic direct xenon lamps and impulse direct xenon lamps for optical laser pumping was built. The accuracy of calculations made within the model being considered is approximately $90 \%$. Thus, the built and tested model may be used for applied calculations and modelling operating modes of devices in which non-stationary arc electrical discharges are used, including - for optimization of operating modes of impulse direct xenon lamps.

\section{REFERENCES}

[1] I.D. Kaganovich, V.I. Demidov, S.F. Adams and Y. Raitses, "Nonlocal collisionless and collisional electron transport in low-temperature plasma (invited paper)", Plasma Physics and Controlled Fusion, vol. 51, p. 124003, 2009.

[2] A.A. Kudryavtsev, A.S. Smirnov, L.D. Tsendin, Glow discharge physics. SPB: Lan', 2010.

[3] A. Chirtsov, A. Kudryavtsev, E. Bogdanov, "Fluxes of Charged Particles in Two-Chamber ICP Discharge in Oxygen", Accepted to EEE Transactions on Plasma Science Special Issue, Images in Plasma Science, v.39, N8, P. 2562-2563, 2011.

[4] E.A. Bogdanov, A.S. Chirtsov, A.A. Kudryavtsev, "Fundamental non-ambipolarity of electron fluxes in 2D plasmas", Phys. Rev. Lett., Vol. 106, pp. 195001, 2011.

[5] K. Kapustin, A. Kudryavtsev, E. Bogdanov, A. Chirtsov, "Nonlocal behavior of electron fluxes and excitation rates for "local" EEDF in moderate and high pressures DC positive column plasmas", Imag. Plasma Sci., Vol. 39, pp.180-183, 2011.

[6] S.I. Eliseev, V.I. Demidov, A.A. Kudryavtsev, V.I. Kolobov, E.A. Bogdanov, A.S. Chirtsov, "Impulse breakdown modelling in helium using adaptive methods", J. Sci.-techn. Bull. Inf. Techn., Mech. Opt., Vol. 5, pp.139-146, 2014.

[7] A.S. Chirtsov, V.M. Mikushev, E.V. Lebedeva, S.V. Sychov, "Numerical Simulation of Glow Discharge in Air Mixtures under Low Pressure Conditions", Int. J. Appl. Eng. Res., Vol. 11, pp. 11836-11846, 2016.

[8] H. Mahadevan, L.L.Raja, "Simulations of direct-current airglow discharge at pressures 1 Torr: discharge model validation", J. Appl. Phys., Vol. 107, pp. 093304, 2010.

[9] T. Makabe, T. Tatsumi, "Workshop on atomic and molecular collision data for plasma modelling: Database needs for semiconductor plasma processing", Plasma Sources Science and Technology, vol. 20, n.2, 2011. https://doi.org/10.1088/09630252/20/2/024014.

[10] L.A. Weinstein, I.I. Sobelman, E.A. Yukov. Atom excitation and widening of spectral lines. M.: Science, 1979.

[11] S.V. Sychov, A.S. Chirtsov "Genetic algorithm as a means of solving a radial Schrödinger equations system" presented at 19th International Conference on Soft Computing and Measurements, SCM, St. Petersburg, Russia, 2016
[12] M.V. Chernysheva, A.S. Chirtsov, D.A. Shvager, "Comparative analysis of plasma chemical models for computer simulation of glow discharges in air mixtures", Sci.-Techn. Bull. Inf. Techn., mech. Opt., Vol. 16, pp. 903-916, 2016.

[13] Jr. De Joseph "Non-local effects in a bounded low-temperature plasmas with fast electrons", Phys. Plasmas, Vol. 14, pp. 057101, 2007.

[14] E.A. Bogdanov, K.D. Kapustin, A.A. Kudryavtsev, A.S. Chirtsov, "Comparison of various options of hydrodynamic (fluid) modelling of a longitudinal structure of atmospheric pressure micro-discharge in helium”, Techn. Phys. J., Vol. 80, pp. 41-53, 2010.

[15] E.A. Bogdanov, A.A. Kudryavtsev, N. Ochikova, "Violation of Boltzmann distribution for plasma electron density in twochamber ICP-discharges", Techn. Phys. J., Vol. 85, pp. 155-158, 2015.

[16] A.S. Chirtsov, S. Eliseev, A. Kudryavtsev, H.N. Liu, Y.D. Zhongxi, "Transition from Glow Microdischarge to Arc Discharge with Thermionic Cathode in Argon at Atmospheric Pressure", IEEE Trans. Plasma Sci. Special Iss. - Atmosph. Press. Plasmas Appl., Vol. 44, pp. 2536-2544, 2016.

[17] Comsol 4.0a Plasma module user guide // HUMUSOFT [Website] [Online]. URL: https://www.humusoft.com/comsol/ (Accessed January 2019).

[18] S.I. Eliseev, V.I. Demidov, A.A. Kudryavtsev, V.I. Kolobov, E.A. Bogdanov, A.S. Chirtsov, "Impulse breakdown modelling in helium using adaptive methods", J. Sci.-techn. Bull. Inf. Techn., Mech. Opt., Vol. 5, pp. 139-146, 2014.

[19] ILC Engineering Note No. 152 (Use of Xenon Short Arcs as Pulsed Light Sources), ILC Technology, 399 West Java Drive, Sunnyvale, CA 94089.

[20] G.N. Rokhlin. Discharge light sources. Moscow: Energoatomizdat, 1991.

[21] Xenon Flash Lamps, Technical Literature TLSX1008E04, Hamamatsu Photonics K.K., 1998.

[22] Super Quiet Xenon Lamps, Technical Literature TLSX1002E06, Hamamatsu Photonics K.K, 2000.

[23] W. Koechner Solid-State Laser Engineering. Sixth revised and updated edition. Germany:Springer, 2006.

[24] A.A. Mak, N.L. Soloviev. Introduction in high-temperature laser plasma physics. Leningrad: LSU, 1991.

[25] W.J. O'Brian, G.L. Hunter, J.J. Rosson, R.A. Hulsey, K.E. Carns, "Ultraviolet system design: past, present and future", in : Proceedings Water Quality Technology Conference, AWWA, New Orleans, LA., 1995, pp. 271-305.

[26] J. Dunn, A. Bushnell, W. Ott. \& Clark, "Pulsed white light food processing", Cereal Foods World, Vol. 42, pp. 510-515, 1997.

[27] J. Dunn, D. Burgess, F. Leo, "Investigation of pulsed light for terminal sterilization of WFI filled blow/fill/seal polyéthylène containers. Parenteral Drug Assoc.”, J. Pharm. Sci. \& Tech., Vol. 51, pp. 111-115, 1997.

[28] High-voltage engineering. Gas discharge process physics. St.Petersburg: SPBSTU, 1999.

[29] S.K. Zhdanov, V.A. Kurnaev et al. Fundamentals of physical processes in plasma and plasma units. Moscow: MSIPI, 2000.

[30] A. G. Grigoryants, I. N. Shiganov. A. M. Chirkov. Hybrid laser welding technologies. Moscow: Publ. Bauman's MSTU, 2004.

[31] A. Mimouni, "Inactivation microbienne par lampes flash ou lumière pulsée", La Lettre - Traitements de surfaces, Vol. 10, pp. 21-25, 2004.

[32] F. Fine, P. Gervais, "Efficiency of pulsed UV light for microbial decontamination of food Powders", J. Food Protection, Vol. 67, pp. 787-792, 2004.

[33] B. Mrabet, H. Elloumi, A. Chammam, M. Stambouli, G. Zissis, "Effect of a pulsed power supply on the ultraviolet radiation and electrical characteristics of low pressure mercury discharge" Plasma Devices and Operations, Vol.14, pp. 249-259, 2006.

[34] L. Bouslimi, A. Chammam, M. Ben Mustapha, M. Stambouli, J.P. Cambronne, "Simulation and Experimental Study of an Electronic Pulsed Power Supply for HID Lamps Intended for Photochemical Applications", Int. Rev. Electrical Eng. (IREE), Vol. 4, pp. 799808, 2009.

[35] L. Bouslimi, A. Chammam, M. Ben Mustapha, M. Stambouli, J.P. Cambronne, "Electric and spectral characterization of a high pressure mercury lamp used in the photochemical treatment", Int. J. Sci. Techn. Automatic Control Comp. Eng. (IJ-STA), Vol. 3, pp. 1064-1071, 2009.

[36] I.S. Marshak. Pulse light sources. Moscow: Energiya, 1978.

[37] Y.A. Mandryko, "Electrical circuit with switching IGBTtransistors for gas discharge lamps", Adv. Electronics, Vol. 7, pp. 64-69, 2015. 